\title{
Saúde ocupacional de profissionais de enfermagem e a depressão
}

\author{
Occupational health of nursing professionals and depression \\ Salud ocupacional de profesionales de enfermería y depresión
}

Recebido: 04/06/2021 | Revisado: 08/06/2021 | Aceito: 17/06/2021 | Publicado: 02/07/2021

\author{
Aline de Oliveira Novato Moraes \\ ORCID: https://orcid.org/0000-0002-3452-3921 \\ Centro Universitário de Volta Redonda, Brasil \\ E-mail: ninne.vr@gmail.com \\ Carolyne da Silva Martins Barreto \\ ORCID: https://orcid.org/0000-0002-9265-3994 \\ Centro Universitário de Volta Redonda, Brasil \\ E-mail: carolyne-barreto@hotmail.com \\ Mayara Gonçalves dos Santos \\ ORCID: https://orcid.org/0000-0001-5687-2025 \\ Centro Universitário de Volta Redonda, Brasil \\ E-mail: mayvrji@gmail.com \\ Clarissa Ferreira Pontual de Oliveira \\ ORCID: https://orcid.org/0000-0002-2915-9205 \\ Centro Universitário de Volta Redonda, Brasil \\ E-mail: pontualclarissa@gmail.com \\ Renata Martins da Silva Pereira \\ ORCID: https://orcid.org/0000-0001-7642-6030 \\ Centro Universitário de Volta Redonda, Brasil \\ Universidade do Estado do Rio de Janeiro, Brasil \\ E-mail: renataenfprofessora@gmail.com
}

\begin{abstract}
Resumo
Este estudo teve como objetivos identificar os principais fatores que afetam a saúde ocupacional de trabalhadores de enfermagem desencadeando a depressão e apontar estratégias que podem ser utilizadas para lidar com o estresse ocupacional, evitando assim a depressão. Pesquisa bibliográfica, exploratória, descritiva, com uma abordagem qualitativa, tendo como aporte teórico a Teoria das Necessidades Humanas Básicas de Maslow (1954). O levantamento dos artigos foi realizado na Biblioteca Virtual em Saúde (BVS) nas bases de dados: Lilacs, BDENF, Medline, Index de Psicologia - Periódicos Técnico-Científicos e Campus Virtual de Saúde Pública Brasil. Os descritores utilizados foram: "saúde ocupacional", "enfermagem" e "depressão", cruzados entre si. Selecionou-se 10 artigos desenvolvidos entre os anos de 2002 a 2018. Os resultados da pesquisa apontaram que os trabalhadores de enfermagem podem desenvolver sinais e sintomas de alterações psíquicas, emocionais e comportamentais, e ainda, com frequência fazem uso de medicamentos psicofármacos sem prescrição e acompanhamento médico. As estratégias que podem auxiliar no controle desses sintomas são a gestão de pessoal, apoio dos líderes, reuniões para exposição de dificuldades cotidianas, solicitação de avaliação da medicina do trabalho e uso da teoria das necessidades humanas básicas de Maslow para auxílio na identificação de problemas. Concluiu-se que a depressão é uma doença mental caracterizada pelo desequilíbrio químico nas células cerebrais e afeta significativamente a qualidade de vida no trabalho dos membros da equipe de enfermagem; dessa forma, os líderes das equipes de enfermagem devem estar atentos aos sinais de adoecimento físico e psíquico de seus trabalhadores para assim ajudar os mesmos.
\end{abstract}

Palavras-chave: Enfermagem; Saúde ocupacional; Depressão.

\begin{abstract}
This study aimed to identify the main factors that affect the occupational health of nursing workers that trigger depression and point out strategies that can be used to deal with occupational stress, thus avoiding depression. Bibliographic research, exploratory, descriptive, with a qualitative approach, having as theoretical support the Theory of Basic Human Needs by Maslow (1954). The survey of articles was carried out in the Virtual Health Library (VHL) in the following databases: Lilacs, BDENF, Medline, Psychology Index - Technical-Scientific Periodicals and Virtual Campus of Public Health Brazil. The descriptors used were: "occupational health", "nursing" and "depression", crossed with each other. 10 articles were selected between the years 2002 to 2018. The research results showed that nursing workers can develop signs and symptoms of psychological, emotional and behavioral changes, and also often use over-the-counter psychotropic medications and medical follow-up. Strategies that can help control these symptoms are personnel management, support from leaders, meetings to expose daily difficulties, request for an
\end{abstract}


occupational medicine assessment, and use of Maslow's theory of basic human needs to help identify problems. It was concluded that depression is a mental illness characterized by chemical imbalance in brain cells and significantly affects the quality of life at work of nursing team members; thus, the leaders of the nursing teams must be aware of signs of physical and psychological illness in their workers, in order to help them.

Keywords: Nursing; Occupational health; Depression.

\section{Resumen}

Este estudio tuvo como objetivo identificar los principales factores que afectan la salud ocupacional de los trabajadores de enfermería que desencadenan la depresión y señalar estrategias que se pueden utilizar para enfrentar el estrés ocupacional, evitando así la depresión. Investigación bibliográfica, exploratoria, descriptiva, con enfoque cualitativo, teniendo como soporte teórico la Teoría de las Necesidades Humanas Básicas de Maslow (1954). La encuesta de artículos se realizó en la Biblioteca Virtual en Salud (BVS) en las siguientes bases de datos: Lilacs, BDENF, Medline, Índice de Psicología - Revistas Técnicas-Científicas y Campus Virtual de Salud Pública Brasil. Los descriptores utilizados fueron: "salud ocupacional", "enfermería" y "depresión", cruzados entre sí. Selecionou-se 10 artigos desenvolvidos entre os anos de 2002 a 2018. Os resultados da pesquisa apontaram que os trabalhadores de enfermagem podem desenvolver sinais e sintomas de alterações psíquicas, emocionais e comportamentais, e ainda, com frequência fazem uso de medicamentos psicofármacos sem prescrição e supervisión médica. Las estrategias que pueden ayudar a controlar estos síntomas son la gestión de personal, el apoyo de los líderes, las reuniones para exponer las dificultades diarias, la solicitud de una evaluación de medicina ocupacional y el uso de la teoría de Maslow de las necesidades humanas básicas para ayudar a identificar problemas. Se concluyó que la depresión es una enfermedad mental caracterizada por un desequilibrio químico en las células cerebrales y que afecta significativamente la calidad de vida en el trabajo de los miembros del equipo de enfermería; así, los líderes de los equipos de enfermería deben estar atentos a los signos de enfermedad física y psicológica en sus trabajadores, para poder ayudarlos.

Palabras clave: Enfermería; Salud laboral; Depresión.

\section{Introdução}

A saúde ocupacional ou saúde do trabalhador diz sobre o bem-estar do mesmo em seu ambiente de trabalho, como promoção e preservação da integridade física e emocional durante o exercício da função, buscando sempre detectar fatores de risco ao seu bem-estar (Carlotto, Câmara, Braun, Rodriguez \& Diehl, 2017).

Nos cenários do cuidar, há fatores que podem gerar uma exaustão física e psíquica no trabalhador de enfermagem, como: sobrecarga de trabalho, carga horária exaustiva, falta de autonomia e controle dos processos, presença de riscos físicos, químicos e biológicos, o lidar com sofrimento, a insuficiência de recursos, a responsabilidade com vidas, dentre outros (Ribeiro, Marchi, Marins \& Gonçalves, 2020).

Tais fatores afetam severamente a saúde e o bem-estar dos enfermeiros e sua equipe; causando inicialmente o estresse ocupacional, que quando não tratado pode levar ao surgimento de doenças físicas, psíquicas, emocionais e comportamentais. Dentre essas doenças que podem surgir, a depressão vem acometendo uma grande quantidade de membros da equipe de enfermagem que atuam nos diversos locais de assistência à saúde. A depressão é uma doença psiquiátrica e crônica, caracterizada pela perda de interesse e prazer à vida, submetendo o ser a um desânimo profundo e tornando-o desesperançoso. Não há especificidade na causa da doença.

Sabe-se, porém, que alterações químicas nas células cerebrais acontecem quando um indivíduo se encontra em estado de tristeza. Caso este esteja propenso, como por exemplo, tenha um histórico familiar de depressão, pode desenvolvê-la, ratificando, entretanto que, não há uma causa bem definida para a mesma (Tenório, 2017).

Entre os trabalhadores da saúde, os profissionais de enfermagem estão no grupo dos mais propensos aos problemas de saúde mental, dentre eles depressão e o risco de suicídio, porque lidam com o sofrimento humano, a dor, a alegria, a tristeza e necessitam ofertar ajuda àqueles que necessitam de seus cuidados. $\mathrm{O}$ aparecimento destas patologias encontra-se relacionado à vida pessoal e a vida profissional dos indivíduos, e o fato de estar em constante posição de ajudar o outro, ser o porto-seguro, bom ouvinte e compreensivo, ainda que involuntariamente, faz com que torne grupo alvo de acometimento de problemas de saúde mental, a saber a depressão (Barbosa, Belasco \& Rios, 2010). 
Destacam-se, ainda, outros fatores comumente encontrados, como as condições difíceis de trabalho e a falta de reconhecimento profissional (Silva, et.al., 2015).

A depressão não se manifesta rapidamente no profissional.

Primeiro são encontrados estresses elevados que quando não tratados e resolvidos, causam deterioração da saúde mental, que podem se manifestar por depressão ou até mesmo Síndrome de Burnout (Vasconcelos, Martino \& França, 2018).

A atenção reduzida, possível lentidão mental e raciocínio em consequência de uma depressão já instalada, geram um declínio nos serviços prestados pelo profissional. Com isso, os riscos de acidentes laborais típicos da área aumentam, ocasionando riscos para a vida do profissional e do paciente (Melo, et.al., 2021).

Estudos que buscam conhecer a produção bibliográfica acerca de saúde ocupacional de profissionais de enfermagem e a depressão torna-se relevante, pois segundo a Organização Mundial de Saúde, os transtornos mentais comuns apresentam 13\% do total de todas as doenças e atingem cerca de 700 milhões de pessoas no mundo, apresentando diversos tipos de sinais e sintomas sendo que os mais prevalentes são depressão, ansiedade e estresse, sendo estimado para 2020 que a depressão seja a segunda maior causa de incapacitação da população mundial (Aguiar, 2017).

Surge assim, como questão a investigar na pesquisa:

- O que a literatura revela acerca da saúde ocupacional de trabalhadores de enfermagem e o surgimento da depressão?

Objetivou-se com esta pesquisa os principais fatores que afetam a saúde ocupacional de trabalhadores de enfermagem desencadeando a depressão e apontar estratégias que podem ser utilizadas por trabalhadores de enfermagem para lidar com o estresse ocupacional, evitando assim a depressão.

\section{Metodologia}

O estudo realizado consiste em uma pesquisa bibliográfica, exploratória, descritiva, com uma abordagem qualitativa.

Michel (2015) define a pesquisa qualitativa, como aquela que se propõe a colher e analisar dados descritivos, obtidos diretamente da situação estudada; enfatiza o processo mais que o resultado, para o que precisa e retrata a perspectiva dos participantes. Na pesquisa qualitativa, verifica-se a realidade em um contexto natural, tal como ocorre na vida real, procurando dar sentido aos fenômenos ou interpretá-los, de acordo com os significados que possuem para as pessoas implicadas nesse contexto (Michel, 2015).

O levantamento dos artigos foi realizado na Biblioteca Virtual em Saúde (BVS) que integra as seguintes bases de dados: Literatura Latino-Americana e do Caribe em Ciências da Saúde (Lilacs), Base de Dados de Enfermagem (BDENF), Medical Literature Analysis and Retrieval SystemOnline (Medline), Index de Psicologia - Periódicos Técnico-Científicos e Campus Virtual de Saúde Pública Brasil.

A estrutura da pesquisa em cada base de dados foi conduzida por meio dos descritores controlados pelo DeCS: "saúde ocupacional", "enfermagem" e "depressão", cruzados entre si. Vale ressaltar que o período de publicação foi delimitado nesta fase, incorporando os anos de 2002 a 2018. Foram encontradas 187 produções científicas e após a utilização dos seguintes filtros: texto completo e disponível, idioma português e assunto principal: depressão.

Selecionou-se 10 artigos.

A etapa de seleção dos estudos envolveu a leitura crítica e atenta dos artigos na íntegra, aplicando os seguintes critérios: 1) Inclusão - estudos originais, publicados no idioma português nos últimos 16 anos, que abordassem o conceito da saúde ocupacional da equipe de enfermagem e a depressão. 2) Exclusão - não atendesse aos critérios de inclusão e estarem duplamente indexados nas bases. A coleta de dados deu-se no período do mês de novembro de 2019. 
Inicialmente foi feita uma leitura flutuante dos artigos selecionados, e logo em seguida foi realizada uma leitura analítica dos artigos, realizando a interpretação dos dados. Após a interpretação dos dados, foi possível construir as seguintes categorias temáticas: fatores que desencadeiam a depressão, sinais e sintomas da depressão, uso/abuso de medicamentos e estratégias para lidar com o estresse ocupacional.

Para iluminar os achados deste estudo, optamos por abordar a Teoria das Necessidades Humanas Básicas de Abraham Maslow (1954). Segundo Maslow, o homem é motivado segundo suas necessidades que se manifestam em graus de importância onde as fisiológicas são as necessidades iniciais e as de realização pessoal são as necessidades finais. Cada necessidade humana influencia na motivação e na realização do indivíduo que o faz prosseguir para outras necessidades que marcam uma pirâmide hierárquica (Schultz \& Schultz, 2016).

As necessidades fisiológicas se encontram como base para a pirâmide, segundo Maslow. As necessidades de segurança aparecem após o suprimento das necessidades fisiológicas. As necessidades sociais somente aparecerão após as necessidades de segurança serem supridas. As necessidades de status e estima ocorrem depois que as necessidades sociais são supridas. As necessidades de autorrealização se encontram no topo da pirâmide hierárquica (Schultz \& Schultz, 2016).

Nesse estudo, busca-se relacionar as necessidades humanas básicas dos membros de enfermagem, com a saúde ocupacional dos mesmos. Quando as necessidades básicas dos trabalhadores de enfermagem ficam comprometidas no cotidiano de trabalho, podem surgir insatisfações na relação do cuidado com o outro e na relação com o ambiente. Isso pode gerar comprometimentos físicos, psíquicos, emocionais e comportamentais nos profissionais, que quando não identificados precocemente e tratados podem desencadear a patologia depressão.

A pirâmide de Maslow foi adaptada ao ambiente de trabalho, com vistas em melhor entender como as necessidades humanas básicas se expressam no ambiente profissional e nos trabalhadores que ali se encontram. A satisfação das necessidades humanas básicas implica em trabalhadores mais motivados, isso traz consequências positivas para a instituição com redução de custos, otimização de processos e diminuição da rotatividade dos recursos humanos.

\section{Resultados e Discussão}

Como resultado obteve-se das bases de dados utilizadas nesta pesquisa.

Quadro 1 - Distribuição dos estudos sobre Saúde Ocupacional de Trabalhadores de Enfermagem e a Depressão, segundo título, revista, ano e objetivos. Volta Redonda/ RJ, 2020.

\begin{tabular}{|l|l|l|l|}
\hline \multicolumn{1}{|c|}{ Título } & \multicolumn{1}{|c|}{ Revista } & Ano & \multicolumn{1}{|c|}{ Objetivos } \\
\hline $\begin{array}{l}\text { 1) Sintomas depressivos e uso de } \\
\text { drogas entre profissionais da } \\
\text { equipe de enfermagem }\end{array}$ & Escola Ana Nery & 2018 & $\begin{array}{l}\text { Avaliar possíveis relações entre abuso de drogas, sintomas } \\
\text { sugestivos de depressão e gênero em profissionais da equipe de } \\
\text { enfermagem. }\end{array}$ \\
\hline $\begin{array}{l}\text { 2) Sintomas de depressão e e } \\
\text { fatores intervenientes entre } \\
\text { enfermeiros do serviços } \\
\text { hospitalar de emergência }\end{array}$ & $\begin{array}{l}\text { Acta Paulista de } \\
\text { Enfermagem }\end{array}$ & 2015 & $\begin{array}{l}\text { Verificar se enfermeiros do serviço hospitalar de emergência } \\
\text { apresentavam identificar fatores } \\
\text { intervenientes e avaliar percepção sobre o sofrimento psíquico e } \\
\text { influência na assistência prestada }\end{array}$ \\
\hline $\begin{array}{l}\text { 3) Evidência de validade } \\
\text { preliminar da escala de depressão } \\
\text { (EDEP): Um estudo com alunos } \\
\text { de enfermagem }\end{array}$ & $\begin{array}{l}\text { Psicologia } \\
\text { Argumentada }\end{array}$ & 2014 & $\begin{array}{l}\text { Buscar evidências baseando-se na relação com outras variáveis de } \\
\text { uma versão de 32 itens da EDEP correlacionando-as com a Escala } \\
\text { de Vulnerabilidade ao Estresse no Trabalho. }\end{array}$ \\
\hline $\begin{array}{l}\text { 4) Adoecimento psíquico de } \\
\text { trabalhadores de Unidades de } \\
\text { Terapia Intensiva }\end{array}$ & $\begin{array}{l}\text { Psicologia Ciência e } \\
\text { Profissão }\end{array}$ & 2013 & $\begin{array}{l}\text { Compreender os aspectos da organização do trabalho que podem } \\
\text { estar associados ao adoecimento psíquico em trabalhadores da } \\
\text { saúde de UTI; Estratégias defensivas utilizadas e utilização do }\end{array}$ \\
\hline
\end{tabular}




\begin{tabular}{|c|c|c|c|}
\hline & & & método misto. \\
\hline $\begin{array}{l}\text { 5) Impacto de um programa de } \\
\text { atividade física sobre a } \\
\text { ansiedade, depressão, estresse } \\
\text { ocupacional e síndrome de } \\
\text { burnout dos profissionais de } \\
\text { enfermagem no trabalho }\end{array}$ & $\begin{array}{l}\text { Revista } \\
\text { Americana } \\
\text { Enfermagem }\end{array}$ & 2014 & $\begin{array}{l}\text { Avaliar os efeitos de um programa de atividade física no local de } \\
\text { trabalho sobre os níveis de ansiedade, depressão, Burnout, estresse } \\
\text { ocupacional e na autopercepção da saúde e qualidade de vida, } \\
\text { relacionada ao trabalho de uma equipe de enfermagem de } \\
\text { cuidados paliativos. }\end{array}$ \\
\hline $\begin{array}{l}\text { 6) Fatores associados á depressão } \\
\text { relacionada ao trabalho de } \\
\text { enfermagem }\end{array}$ & Estudos de Psicologia & 2007 & $\begin{array}{l}\text { Identificar evidências científicas sobre a ocorrência de depressão } \\
\text { relacionada ao trabalho de enfermagem e caracterizar fatores } \\
\text { desencadeantes e estratégias utilizadas para a prevenção de doença }\end{array}$ \\
\hline $\begin{array}{l}\text { 7) Qualidade vida e sintomas } \\
\text { depressivos em residentes de } \\
\text { enfermagem }\end{array}$ & $\begin{array}{l}\text { Revista } \\
\text { Americana } \\
\text { Enfermagem }\end{array}$ & 2005 & $\begin{array}{l}\text { Avaliar a qualidade de vida e a prevalência de sintomas } \\
\text { depressivos nos residentes de enfermagem de uma Escola Paulista } \\
\text { de Medicina }\end{array}$ \\
\hline $\begin{array}{lcc}\text { 8) Depressão } & \text { no trabalho } & \text { de } \\
\text { enfermagem: } & \text { Revisão } & \text { de } \\
\text { literatura } & & \end{array}$ & $\begin{array}{l}\text { Universistas: Ciências } \\
\text { da Saúde, Brasília }\end{array}$ & 2015 & $\begin{array}{l}\text { Identificar estudos que analisaram a ocorrência de depressão } \\
\text { relacionada ao trabalho de enfermagem, a fim de nortear } \\
\text { estratégias de prevenção e enfrentamento junto a esses } \\
\text { profissionais. }\end{array}$ \\
\hline $\begin{array}{l}\text { 9) Causa de afastamento entre } \\
\text { trabalhadores de enfermagem de } \\
\text { um hospital público do interior } \\
\text { de São Paulo. }\end{array}$ & $\begin{array}{lr}\text { REME } \quad \text { Revista } \\
\text { Mineira } & \text { de } \\
\text { Enfermagem } & \end{array}$ & 2005 & $\begin{array}{l}\text { Identificar as características dos trabalhadores de enfermagem que } \\
\text { se afastaram do trabalho no período de um ano e quais as causas } \\
\text { que levaram ao afastamento }\end{array}$ \\
\hline $\begin{array}{l}\text { 10) Estresse ocupacional no } \\
\text { trabalho e enfermagem no Brasil: } \\
\text { Uma revisão integrativa }\end{array}$ & $\begin{array}{l}\text { Revista Bras Promoção } \\
\text { Saúde - Fortaleza }\end{array}$ & 2016 & $\begin{array}{l}\text { Descrever os fatores desencadeadores do estresse ocupacional em } \\
\text { profissionais de enfermagem, bem como os riscos relacionados } \\
\text { com o desenvolvimento desse estresse. }\end{array}$ \\
\hline
\end{tabular}

Fonte: Autores.

\section{Fatores que Desencadeiam a Depressão}

Ao analisarmos os artigos científicos identificados, percebeu-se que o ambiente de trabalho pode gerar fatores que desencadeiam sofrimento emocional e psíquico dos membros da equipe de enfermagem. Os trechos abaixo revelam esses fatores:

O ambiente de trabalho na Enfermagem apresenta estressores importantes a influenciar a qualidade de vida dos profissionais e são fatores predisponentes ao adoecimento, sendo relevante a pesada carga de trabalho, salário insuficiente, discriminação social, expectativas elevadas, grau elevado de responsabilidade para com os pacientes e a atmosfera física. Artigo 2

Os dados mostraram que 30,2\% dos enfermeiros relataram a sobrecarga de trabalho, o baixo salário, carga horária excessiva, desgaste, preocupação com o trabalho como responsáveis pelo desenvolvimento de seu quadro depressivo. Artigo2

Em sua prática diária esses profissionais ao prestarem assistência direta ao paciente estão expostos a vários riscos correlacionados à atividade laboral que podem ser acometidos por fatores intrínsecos e extrínsecos como os riscos: químicos, físicos, mecânicos, biológicos e ergonômicos que podem causar agravos ocupacionais. Artigo 10

(...) as pressões no trabalho, como conflito de interesse e a sobrecarga, contribuem para o desequilíbrio e estresse, que levam à deterioração da saúde mental manifestada principalmente pela depressão. Artigo 8

No setor de saúde, os profissionais estão expostos aos riscos físicos, químicos, biológicos, ergonômicos (mecânicos), psicossociais e de acidente. Devido a essas peculiaridades, a exposição contínua e múltipla pode desenvolver o adoecimento dos trabalhadores, acarretando prejuízos às instituições de saúde empregadoras e as instituições governamentais, podendo 
interferir na qualidade da assistência prestada aos clientes, uma vez que, o estado de saúde do trabalhador interfere diretamente no desenvolvimento das suas atividades laborais (Almeida, Torres \& Santos, 2012).

Em pesquisas realizadas em vários países, nota-se que a enfermagem tem longas jornadas de trabalho, associadas a más condições laborais, pouca autonomia, muita responsabilidade e baixa valorização, que acarretam a uma elevada demanda física e emocional. Essas situações têm contribuído para a perda parcial ou total das capacidades corporais e psíquicas desses profissionais, com prejuízos na vida cotidiana em razão da exaustão física e da emocional, que se apresentam de modo mais frequente em enfermeiros do que entre a população em geral (Oliveira, Silva \& Lima, 2018).

Quando aplicamos a pirâmide de Maslow ao ambiente de trabalho, percebe-se que: na base se encontra o descanso físico e mental, salário suficiente, disponibilidade de horários para alimentação e pausas durante o expediente; logo em seguida a garantia de estabilidade, bom salário, ambiente de trabalho seguro e livre de acidentes; depois as boas relações com lideranças e pares, sentir-se acolhido pelas pessoas da instituição; em seguida ser reconhecido pelos seus resultados, ter aumentos de salários, ter sua opinião como profissional respeitada; e solução de problemas no dia a dia (Schultz \& Schultz, 2016).

Os trabalhadores de enfermagem necessitam cuidar da sua saúde física e psíquica, considerando o fato de estarem continuamente expostos a fatores que afetam sua qualidade de vida no trabalho. Quando esses profissionais não criam estratégias para lidar com esses fatores, podem desenvolver estresse ocupacional, que quando não tratado, leva em último estágio uma depressão.

\section{Sinais e Sintomas da Depressão}

A análise dos artigos pesquisados possibilitou-nos ainda identificar que os trabalhadores de enfermagem podem apresentar sinais e sintomas significativos de sofrimento psíquico inicialmente, e se não tratado, pode evoluir para diversas patologias, em destaque a depressão. Como visto nos parágrafos abaixo:

A Escala de Depressão apresenta 21 indicadores de depressão, a saber: humor deprimido, inutilidade, autocrítica exacerbada, falta de perspectiva do presente, anedonia, irritabilidade, lentidão psicomotora, queda de produtividade, autoestima rebaixada, perda da libido, incapacidade, alteração do sono, desesperança, ideação suicida, esquiva de situações sociais, indecisão, fadiga, perda de energia, inadequação, esquiva, desamparo. Artigo 3

Um estudo de revisão da literatura sobre burnout em base de dados nacionais e internacionais, entre os anos 1985 e 2006, indicou que essa síndrome apresenta frequentemente Depressão associada, como comorbidade. Esta é considerada na Classificação Estatística Internacional de Doenças e Problemas Relacionados à Saúde, CID - 10 (1993), um transtorno do humor, em que a perturbação fundamental é uma alteração do humor ou do afeto, perda de interesse e de prazer, e em que a energia é reduzida, o que leva a um cansaço aumentado e a atividade diminuída. Outros sintomas comuns nessa patologia são: concentração e atenção reduzidas, arrefecimento na autoestima e na autoconfiança, ideias de culpa e de inutilidade, visões desoladas e pessimistas do futuro, ideias, atos autolesivos ou suicídio, sono perturbado e apetite diminuído. Artigo 4

(...) A mais frequente forma clínica assumida por esse transtorno é geralmente denominada Depressão, envolvendo uma série de sintomas como tristeza, autodepreciação, abandono, desvalia, culpa, entre tantos outros afetos. Artigo 3

A falta de interesse, lentidão nas atividades, inquietação, cansaço, redução da energia, apatia, dificuldade de concentração, pensamento negativo e recorrente, com perda da capacidade de planejamento e alteração do juízo de verdade são evidências de sofrimento humano que sinalizam para depressão e possível risco de suicídio (Silva,et. al., 2015).

As características sintomatológicas de um episódio depressivo maior, segundo o Manual de Diagnóstico e Estatística dos Transtornos Mentais, podem ser resumidas em humor deprimido, perda de interesse ou prazer, problemas psicomotores, de concentração, sono, apetite, fadiga, sentimento de inutilidade ou culpa (Gomes \& Oliveira, 2013). 
Os sinais e sintomas de uma depressão nem sempre são evidentes e, às vezes, são muito bem mascarados pelos indivíduos. Por isso a necessidade da sensibilização dos líderes na enfermagem, para estarem prontos para identificar esses sinais e sintomas, e oferecer auxílio o mais rápido possível aos trabalhadores para que não haja agravamento da patologia e um possível suicídio.

\section{Uso/Abuso de Medicamentos}

Emergiram ainda na pesquisa trechos de artigos científicos que destacaram que há um uso/abuso de medicamentos psicotrópicos por parte dos profissionais de enfermagem, como visto abaixo:

Pesquisa mostrou que 6,1\% de 49 profissionais de enfermagem de uma Unidade de Terapia Intensiva (UTI) faziam uso de benzodiazepínicos e 4\% usavam analgésicos, sendo que somente em $24,4 \%$ o uso de medicamentos era prescrito. Nos Estados Unidos da América (EUA), de 2439 enfermeiros residentes avaliados em programas de anestesiologia, foi observado que os benzodiazepínicos foram a terceira droga de maior abuso entre esses profissionais. Artigo 1

Novamente, o uso de substâncias psicotrópicas, assim como a depressão, no cotidiano de trabalho da enfermagem, podem estar relacionados às condições de trabalho, às dificuldades em lidar com o sofrimento e a morte, às relações interpessoais e interprofissionais e ao despreparo profissional. O consumo dessas substâncias objetiva diminuir as cargas físicas e psíquicas a que esses profissionais estão continuamente expostos. $O$ uso abusivo de sedativos deve ser seriamente investigado no âmbito da enfermagem, considerando a particularidade do grande contingente de profissionais do sexo feminino e de que se trata da única classe de substâncias psicoativas na qual a taxa de consumo entre mulheres é maior, quando se compara aos homens. Além disso, profissionais do sexo feminino apresentam risco aumentado para o desenvolvimento de dependência dessa droga. Artigo 1

Alguns estudos demonstraram que a incapacidade de enfrentamento dos problemas advindos do trabalho, na área da enfermagem, e o alto nível de estresse ocupacional podem levar os profissionais ao uso de ansiolíticos, na busca ativa para tentarem amenizar e remediar o sofrimento que estão expostos no dia a dia. Artigo 10

Os problemas de saúde gerados nos profissionais devido a fatores estressantes não resolvidos em seu ambiente de trabalho como: cansaço, tensão muscular, nervosismo, irritabilidade, dor lombar, ansiedade, tensão pré-menstrual, cefaleias, problemas de memória, depressão, entre outros, estão relacionados com os motivos que levam esses profissionais na busca de estratégias que reduzam esses fatores no trabalho promovendo a saúde e qualidade de vida do trabalhador. Entre essas estratégias, alguns profissionais têm buscado, de forma abusiva, o uso de psicofármacos, ou seja, drogas lícitas, como forma de aliviar essas situações (Ribeiro, 2019).

Médicos e enfermeiros são os profissionais que tem mais tendências a se tornarem dependentes das drogas psicoativas (Vieira, Brida, Macuchu, Massuda \& Preza, 2016).

É preocupante que profissionais da saúde, em destaque os que compõem a equipe de enfermagem, estejam fazendo uso de medicamentos psicotrópicos sem prescrição e acompanhamento médico. Utilizando medicações que causam sedação, que aliviam as dores e agem no Sistema Nervoso Central, podendo agravar ainda mais essa situação de estresse e dependência frente às questões inerentes ao trabalho.

\section{Estratégias para Lidar com o Estresse Ocupacional}

Os artigos científicos ainda revelaram a necessidade da criação de estratégias por parte de todos da equipe de enfermagem e da equipe de saúde para lidar com o estresse ocupacional, como visto abaixo: 
Acredita-se que o bem-estar dos cuidadores profissionais é importante, para que possam oferecer excelência no atendimento ao paciente. Assim, a busca por intervenções dirigidas a profissionais de enfermagem, em um esforço para reduzir o risco de problemas devidos à saúde ocupacional, é de extrema importância. Uma metanálise mostrou que, dentre outros benefícios, os exercícios físicos ocupacionais podem ser eficazes na redução do estresse ocupacional. Artigo 5

(...) necessidade da implantação de programas de atenção à saúde dos trabalhadores que envolvem grupos de discussão, grupos de vivências, psicoterapia e administração participativa, com utilização de estratégias apropriadas, visando à minimização do estresse e do Burnout, ao gerenciamento da depressão relacionada ao trabalho, à redução do absenteísmo e da rotatividade ente os trabalhadores de enfermagem, o que reforça a necessidade de medidas de acompanhamento, por meio de programas e preventivos e de tratamento. Artigo 8

Uma forma de reverter a situação é reduzir o sofrimento psíquico no ambiente de trabalho associando a uma melhor e mais clara divisão das tarefas entre os trabalhadores de enfermagem e os demais profissionais da saúde, a reposição dos trabalhadores faltantes, o apoio do supervisor e dos colegas quando a solução de problemas na clínica, o reconhecimento por parte dos superiores; a participação no processo de tomada de decisão, a oportunidade para desenvolver suas habilidades; e momentos para falarem sobre as tensões no trabalho (Bissoli, 2017).

A Teoria das Necessidade Humanas Básicas de Maslow (1954) constitui uma ferramenta significativa nesse processo, pois ela auxilia os trabalhadores de enfermagem a realizarem seu auto-conhecimento, principalmente na identificação dos fatores que os motivam no ambiente de trabalho.

A atuação da Medicina do Trabalho, realizando o acompanhamento dos profissionais e avaliação contínua do local de trabalho, desenvolvendo grupos de apoio e a busca ativa daqueles que poderiam estar mais suscetíveis à depressão e sofrimento psíquico, pois acredita-se que a prevenção e o tratamento deveriam ter uma abordagem multidisciplinar, na qual enfermeiro, psicólogo e médico, poderiam estar intervindo para que haja uma melhora na qualidade de vida no trabalho (Antunes, 2017).

É de grande relevância que os ambientes do cuidar em enfermagem possam estar preparados para criar alternativas de alívio das tensões aos profissionais. Os líderes de equipe necessitam realizar reuniões constantes com os trabalhadores, de modo que os mesmos possam falar, serem ouvidos, exporem seus medos e angústias. E juntos, em equipe, possam ser traçadas estratégias efetivas para lidar com o estresse ocupacional na enfermagem.

\section{Conclusão}

Sendo a depressão uma doença mental caracterizada pelo desequilíbrio químico nas células cerebrais, pode-se descrevê-la pela perda de interesse e prazer à vida, tornando o indivíduo desesperançoso, num estado de tristeza profunda. E considerando os fatores geradores de exaustão física e psíquica nos profissionais de enfermagem, devido ao ambiente de trabalho, como: sobrecarga de trabalho, carga horária exaustiva, falta de autonomia e controle dos processos, presença de riscos físicos, químicos e biológicos, o lidar com sofrimento, a insuficiência de recursos, a responsabilidade com vidas, dentre outros, temos este grupo como alvo de adoecimento mental, e como resposta à este acometimento, é possível que os cuidados ofertados aos clientes tornem-se insuficientes, ou até, negligenciados. Como dito acima, não há uma causa pré-definida, sendo apenas uma patologia tratável.

Baseando-se nos pressupostos da Teoria das Necessidades Humanas Básicas de Maslow (1954), observou-se que as principais necessidades dos profissionais de enfermagem são suas condições de trabalho e riscos à saúde. E quando não alcançadas podem gerar situações de sofrimento e adoecimento dos trabalhadores. Os resultados da pesquisa nos permitiram conhecer os principais fatores que podem levar a profissionais de enfermagem a desencadearem estresse ocupacional e mais tarde a depressão, tais como: carga horária excessiva, falta de autonomia, muita responsabilidade e baixa remuneração, falta de reconhecimento social, dificuldades de trabalho em equipe, profissão de ajuda constante etc. 
Foi possível concluir que quando os trabalhadores de enfermagem ficam expostos continuamente a esses fatores laborais estressantes podem desenvolver gradativamente sinais e sintomas de alterações psíquicas, emocionais e comportamentais, podendo se instalar efetivamente a doença depressão.

Concluímos ainda que para lidar com esses sinais e sintomas de alterações físicas e mentais que surgem dentro do processo de trabalho, torna-se necessário lançar mão de estratégias como a gestão de pessoal, apoio dos líderes, reuniões para exposição de dificuldades cotidianas, solicitação de avaliação da medicina do trabalho e uso da teoria das necessidades humanas básicas de Maslow para auxílio na identificação de problemas. E ainda, uma quantidade considerável de profissionais de enfermagem faz uso de medicamentos psicofármacos sem prescrição e acompanhamento médico, devido às facilidades de aquisição dessas medicações.

Portanto, os líderes na enfermagem devem estar atentos aos sinais de adoecimento físico e psíquico de seus trabalhadores, criando estratégias efetivas para prevenir e aliviar os efeitos dos fatores desencadeadores estresse ocupacional, Síndrome de Burnout e a depressão.

\section{Referências}

Aguiar, S. M. de M. T. (2017). Depressão na enfermagem: Uma revisão bibliográfica. http://webcache.googleusercontent.com/search?q=cache:zDmWHZnme6QJ:ufrr.br/enfermagem/index.php\%3Foption\%3Dcom_phocadownload\%26view\%3D category\%26download\%3D299:2017-sonia-maria-de-mello-tavares-depressao-na-enfermagemuma-revisao-bibliografica\%26id\%3D19:trabalho-de-conclusaode-curso\%26Itemid\%3D315+\&cd=2\&hl=pt-BR\&ct=clnk\&gl=br

Almeida, L. G. N., Torres, S. C., \& Santos, C. M. F. dos. (2012).Riscos ocupacionais na atividade dos profissionais de saúde da Atenção Básica.Revista Enfermagem Contemporânea, 1(1), 142-154.

Antunes, M. (2017). Produção Científica Brasileira sobre sofrimento psíquico e depressão da equipe de enfermagem na emergência. Revista Enfermagem Contemporânea, 6(1), 1138.

Barbosa, D. A., Belasco, A. G. S., \& Rios, K. A. (2010). Avaliação de qualidade de vida e depressão de técnicos e auxiliares de enfermagem. Revista LatinoAmericana de Enfermagem, 18(3), 122-130.

Bissoli, A. S. R. (2017). Depressão no profissional de enfermagem: reflexos na assistência prestada. http://repositorio.faema.edu.br/handle/123456789/1173

Carlotto, M. S., Câmara, S. G., Braun, A. C., Rodriguez, S. S., \& Diehl, L. (2017). Psicologia da saúde ocupacional: uma revisão integrativa. Aletheia, 50(1-2), $143-153$.

Franco, G. P., Barros, A. L. B. L., \& Nogueira-Martins, L. A. (2005). Qualidade de vida e sintomas depressivos em residentes de enfermagem. Revista LatinoAmericana de Enfermagem,13(2), 139-144.

Ferreira, L. A. L., \& Ferreira, L. L. (2015). Depressão no trabalho de enfermagem: revisão sistemática de literatura. Universitas: Ciências da Saúde, 13(1), 4148 .

Filho, I. M. M., \& Almeida, R. J. (2016). Estresse ocupacional no trabalho em enfermagem no Brasil: Uma revisão integrativa. Revista Brasileira em Promoção da Saúde, 29(3), 447-454.

Freitas, A. R., Caneseca, E. C., Paiva, C. E., \& Paiva, B. S. R. (2014). Impacto de um programa de atividade física sobre a ansiedade, depressão, estresse ocupacional e síndrome de Burnout dos profissionais de enfermagem no trabalho.Revista Latino-Americana de Enfermagem, 22 (3), $332-336$.

Gomes, R. K., \& Oliveira, V. B. de. (2013). Depressão, ansiedade e suporte social em profissionais de enfermagem.Boletim de Psicologia, 63(138), 023-033.

Gonçalves, J. R. S., Melo, E. P., Lombas, S. R. L., Mariano, C. S., Barbosa, L., \& Chillida, M. S. P. (2005). Causas de afastamento entre trabalhadores de enfermagem de um hospital público do interior de São Paulo. REME- Revista Mineira de Enfermagem, 9(4), 309-314.

Junqueira, M. A. B., Santos, M. A., Araújo, L. B., Ferreira, M. C. M., Giuliani, C. D., \& Pillon. S. C. (2018). Sintomas depressivos e uso de drogas entre profissionais da equipe de enfermagem. Rio de Janeiro, RJ, Escola Anna Nery.

Manetti, M. L., \& Marziale, M. H. P. (2007) Fatores associados à depressão relacionada ao trabalho de enfermagem. Estudos de Psicologia,12(1), 79-85.

Melo, C. C. M., Bernardes, L. F., Morceli, G., Silva, P. G., Pereira, S. S., \& Santos, S. V. M., (2021). Nível de evidência dos estudos relacionados à ansiedade, estresse e depressão dos profissionais de enfermagem. Research, Society and Development, 10(1), e2210111295.

Michel, M. H. (2015). Metodologia e Pesquisa Científica em Ciências Sociais:um guia prático para acompanhamento da disciplina e elaboração de trabalhos monográficos. ( $3^{\mathrm{a}}$ ed.). Editora Atlas.

Monteiro, J. K., Oliveira, A. L. L., Ribeiro, C. S., Grisa, G. H., \& Agostini, N. (2013). Adoecimento Psíquico de trabalhadores de unidades de terapia intensiva. Psicologia Ciência e Profissão, 33(2), 366-379. 
Research, Society and Development, v. 10, n. 7, e55810716831, 2021

(CC BY 4.0) | ISSN 2525-3409 | DOI: http://dx.doi.org/10.33448/rsd-v10i7.16831

Oliveira, B. L.C. A., Silva, A. M. da., \& Lima, S. F. (2018). Carga Semanal de trabalho para enfermeiros no Brasil: desafios ao exercício da profissão.Revista Trabalho, Educação e Saúde, 16(3), 1.221-1.236.

Oliveira, F. P., Mazzaia, M. C., \& Marcolan, J. F. (2015). Sintomas de depressão e fatores intervenientes entre enfermeiros de serviço hospitalar de emergência. Acta Paul Enferm.,11(3), 209-215.

Ribeiro, Í. (2019).Consumo de Substâncias Psicoativas por Trabalhadores de Saúde.Universidade Federal do Piauí-UFPI. Teresina, PI.

Ribeiro, W. A., Marchi, M. V., Marins, A. A., \& Gonçalves, T. A. S. (2020). Fatores de Risco para Depressão no Cotidiano da Equipe de Enfermagem no Âmbito Hospitalar. Research, Society and Development, 9(8), el6985287.

Rueda, F. J. M., Alves, S. M. M., \& Baptista, M. N. (2014). Evidência de validade preliminar da escola de depressão (EDEP): um estudo com alunos de enfermagem. Psicologia Argumento,32(79), 107-117.

Schultz, D. P., \& Schultz, S. E. (2016). História da Psicologia Moderna (10ª ed.). Cengage Learning

Silva, D. dos S. D., Tavares, N. V. da S., Alexandre, A. R. G., Freitas, D. A., Brêda, M. Z., Albuquerque, M. C. dos S. de., \& Neto, V. L. de M. (2015). Depressão e risco de suicídio entre profissionais de Enfermagem: revisão integrativa. Revista da Escola de Enfermagem da USP, $49(6), 1027-1036$.

Tenório, G. (2017). Depressão: sintomas, diagnóstico, prevenção e tratamento. https://saude.abril.com.br/medicina/depressao-sintomas-diagnostico-prevencaoe-tratamento/

Vasconcelos, E. M., Martino, M. M. F. de., \& França, S. P. de S. (2018). Burnout e sintomatologia depressiva em enfermeiros de terapia intensiva: análise de relação. Revista Brasileira de Enfermagem, 71(1), 147-153.

Vieira, G. C. G., Brida, R. L. de., Macuchu, R. da S., Massuda, E. M., \& Preza, G. P. (2016). Uso de psicotrópicos pelo enfermeiro: sua relação com o trabalho. https://online.unisc.br/seer/index.php/cinergis/article/view/8118 